\title{
Transatlantica
}

Revue d'études américaines. American Studies Journal

1 | 2017

Morphing Bodies: Strategies of Embodiment in

Contemporary US Cultural Practices

\section{Colloque international « The Black Metropolis, between Past and Future: Race, Urban Planning and Afro-American Culture in Chicago »}

Université Paris Diderot, Fondation des États-Unis et Sciences Po Paris, 15-18 novembre 2017

Eliane de Larminat et Clément Petitjean

\section{(2) OpenEdition \\ Journals}

Édition électronique

URL : https://journals.openedition.org/transatlantica/8738

DOI : 10.4000/transatlantica. 8738

ISSN : $1765-2766$

Éditeur

Association française d'Etudes Américaines (AFEA)

\section{Référence électronique}

Eliane de Larminat et Clément Petitjean, « Colloque international «The Black Metropolis, between Past and Future: Race, Urban Planning and Afro-American Culture in Chicago » », Transatlantica [En ligne],

1 | 2017, mis en ligne le 29 novembre 2018, consulté le 24 mai 2021. URL : http://

journals.openedition.org/transatlantica/8738; DOI : https://doi.org/10.4000/transatlantica.8738

Ce document a été généré automatiquement le 24 mai 2021.

\section{(c) $(1)$}

Transatlantica - Revue d'études américaines est mise à disposition selon les termes de la licence Creative Commons Attribution - Pas d'Utilisation Commerciale - Pas de Modification 4.0 International. 


\section{Colloque international « The Black Metropolis, between Past and Future: Race, Urban Planning and Afro-American Culture in Chicago »}

Université Paris Diderot, Fondation des États-Unis et Sciences Po Paris, 15-18 novembre 2017

Eliane de Larminat et Clément Petitjean

1 Sous l'égide de l'Université de Chicago, le colloque «The Black Metropolis, between Past and Future: Race, Urban Planning and African-American Culture in Chicago » s'est tenu à Paris les 15 à 18 novembre 2017 entre différents lieux (The University of Chicago Center in Paris, l'Université Paris Diderot, la Fondation des États-Unis et Sciences Po Paris).

2 En l'espace de trois journées et de trois soirées, une vingtaine de communications ont été présentées par des universitaires et des doctorants de différents horizons disciplinaires, civilisationnistes, historiens, sociologues, historiens de l'art, politistes ou encore géographes, mais aussi par des acteurs de la culture ; des films ont été projetés retraçant des expériences quotidiennes, présentes et passées, dans le ghetto noir historique du South Side; et un concert de jazz a rassemblé de jeunes musiciennes et musiciens de Chicago et un duo transnational composé de Mike Reed, batteur de l'Association for the Advancement of Creative Musicians (AACM), et d'Olivier Benoît, guitariste français.

3 Le comité d'organisation du colloque, composé d'Arnaud Collombel, d'Henri Peretz, de Forrest Stuart et de Michael Dawson, est à l'image de la manifestation : international, interdisciplinaire, intergénérationnel.

4 Chaque panel réunit des disciplines des sciences sociales et des humanités autour du projet de penser la richesse et la complexité de l'expérience de Chicago comme métropole noire. En se référant au titre de l'ouvrage incontournable de Horace Cayton et St-Clair Drake, Black Metropolis, le colloque repose la question des définitions 
négatives et positives d'une concentration économique, culturelle et institutionnelle ; il s'agit, avec Cayton et Drake, de revenir sur la construction et la conceptualisation des quartiers noirs de Chicago à la fois comme ghetto et comme Bronzeville (ou espace de rayonnement socio-économique et culturel). Au-delà de la diversité des communications et des évènements proposés, on peut distinguer plusieurs axes de réflexion qui permettent de penser la place des Noirs dans le Chicago d'hier et d'aujourd'hui: le champ politique et les mobilisations sociales; les conditions matérielles d'existence, autour du logement en particulier ; le champ intellectuel et les productions culturelles.

\section{Champ politique et mobilisations}

Dans une conférence remarquée, Michael Dawson brosse un portrait des luttes sociales des Noirs de Chicago aux $\mathrm{XX}^{\mathrm{e}}$ et $\mathrm{XXI}^{\mathrm{e}}$ siècles en les replaçant dans une histoire plus globale du «capitalisme racial». Empruntant ce concept au chercheur marxiste Cedric Robinson, pour qui l'histoire du capitalisme américain est indissociable de l'histoire du système esclavagiste, Dawson montre comment l'intégration nominale des Noirs de Chicago à la sphère économique et au champ politique, laissant penser que leur condition serait plus enviable que celle des Noirs du Sud, masque les processus historiques et sociaux par lesquels l'exploitation de la main d'œuvre s'articule à la racialisation des Noirs et leur relégation structurelle. Dawson identifie trois modes principaux de mobilisation: l'accommodation, symbolisée par la «machine dans la machine " que représentent les quelques aldermen (conseillers municipaux) noirs incarnant modération et soumission au pouvoir politique; l'auto-détermination, incarnée par des organisations comme la Nation of Islam ou le Black Panther Party; l'insurrection, qui correspond aux périodes de forte contestation sociale allant de 1918 à 1940 et de 1960 à 1988.

6 C'est sur cette première période que se penche l'intervention de Davarian Baldwin. Davarian Baldwin met en lumière la dimension internationale, voire internationaliste, des mobilisations des Noirs de Chicago en soutien à l'Éthiopie contre l'invasion fasciste. Qualifiant Chicago de "Vienne de l'antifascisme noir » et rappelant l'importance du Parti communiste américain dans l'organisation de la contestation, Baldwin montre ainsi comment la lutte antifasciste s'articule à une virulente dénonciation du racisme américain et du système "Jim Crow » dans le Sud mais aussi à la ségrégation de fait, aux discriminations à l'embauche et au logement, ou aux expulsions locatives.

7 C'est à des processus similaires de constitution des Noirs en groupe mobilisable et mobilisé que s'intéresse Clément Petitjean, dans une étude portant sur les conditions sociales de production de la "community" à travers une grève de la faim de trentequatre jours organisée à l'été 2015 par une association de quartier contre la fermeture d'un lycée public.

8 Les interventions du journaliste Ethan Michaeli et de l'historien James Grossman abordent sous deux prismes différents l'histoire de la "Grande Migration », processus migratoire qui, en l'espace d'une cinquantaine d'années, du début du $\mathrm{XX}^{\mathrm{e}}$ siècle jusqu'aux années 1970, voit plusieurs millions de descendants d'esclaves quitter le Sud de « Jim Crow » pour les centres industriels du Nord et de l'Ouest. À partir d'anecdotes vivantes, Michaeli revient sur l'histoire du journal noir The Chicago Defender, qui joua un rôle décisif dans la «Grande Migration » et dans la constitution du groupe des Noirs en 
groupe «pour soi ». Prenant le soin de distinguer entre causes et motivations et insistant sur le fait que ces dernières constituent un objet d'étude légitime pour l'historien, James Grossman révèle la place qu'occupait Chicago dans l'imaginaire des Noirs du Sud dans la première moitié du $\mathrm{XX}^{\mathrm{e}}$ siècle et expose la palette de motivations pour faire route vers Chicago. Grossman montre ainsi comment la notion de «place ", à la fois géographique, sociale, économique et politique, était la clé de voûte des relations entre classes sociales et de l'ordre socioéconomique sudiste. Les phénomènes de migration révèlent le refus de nombreux Noirs de rester à la place qui leur a été assignée (" the Negro's place was not in the voting booth») et traduisent leurs aspirations à accéder aussi bien à un plein statut de citoyen qu'à la propriété privée. Selon Grossman, ces aspirations font partie intégrante du phénomène de la "Grande Migration » et doivent être étudiées comme telles.

9 C'est aussi aux aspirations individuelles et collectives, à la fois calculs rationnels et élans émotionnels, qu'Andrew Diamond s'attache dans une communication qui examine la marchandisation de la vie quotidienne dans les années 1930, et ses conséquences en terme de démobilisation politique de la population noire de Chicago sur le long terme. L'intervention d'Andrew Diamond fait ainsi écho aux propos liminaires de Michael Dawson sur l'articulation entre capitalisme racial et phénomènes de mobilisation et de démobilisation politiques.

\section{Conditions matérielles d'existence}

10 Après la communication de Mitch Duneier sur les conditions matérielles et intellectuelles de l'application du terme "ghetto » aux quartiers noirs ségrégués et les débats que suscite ce terme, un ensemble de trois communications s'est penché sur l'histoire du logement, de l'érection de grands ensembles abritant des logements sociaux dans les années 1950 et 1960 à la crise des subprimes du milieu des années 2000 et les terrains vagues qui sont aujourd'hui si caractéristiques de nombreux quartiers noirs, des South et West Sides de Chicago ou d'autres anciens bastions industriels comme Detroit ou Cleveland. Bradford Hunt propose ainsi une interprétation de l'échec des programmes de logements sociaux dans les années 1950 et 1960 et leur rôle majeur dans la reproduction des inégalités sociales : ceux-ci échouent car ils se concentrent davantage sur le bâti que sur ses habitants. Si des travaux pionniers dans ce domaine, comme ceux d'Arnold Hirsch, ont insisté sur le facteur géographique (les projects sont volontairement construits dans des quartiers majoritairement noirs), Hunt fait l'hypothèse que la densité de la population jeune, qu'un faible tissu associatif et institutionnel ne permet pas d'encadrer, a elle aussi contribué à l'échec des programmes. La présentation d'Éliane de Larminat propose une perspective complétant celle de Hunt. En se concentrant sur les évolutions des représentations photographiques des logements sociaux et des rapports de pouvoir entre différentes institutions qui les sous-tendent (Chicago Housing Authority, entreprises médiatiques, associations de quartier), elle montre comment les photographies des «public housing monstrosities » deviennent l'enjeu de luttes symboliques pour la représentation des Noirs. Florence Nussbaum présente ensuite une enquête récente sur la texture visible et invisible de l'abandon résidentiel dans les quartiers pauvres, en exposant les mécanismes de prédation qui permettent de faire discrètement fructifier ces biens aux dépens de la collectivité. 


\section{Champ intellectuel}

11 Le colloque est aussi l'occasion d'esquisser une histoire des sciences sociales et des savoirs scientifiques produits sur (mais aussi depuis) Chicago et la black metropolis. L'ouvrage de Horace Cayton et St-Clair Drake, auquel de nombreux intervenants rendent directement ou indirectement hommage, tisse ainsi un fil rouge tout au long du colloque. Henri Peretz rappelle que des extraits en sont traduits dès 1946 dans la revue Les Temps modernes, qui joua un rôle important dans la mise en circulation des débats intellectuels outre-Atlantique. Derrière Cayton et Drake, c'est le rôle des sociologues de l'Université de Chicago qui se dessine, au premier rang desquels on trouve Robert E. Park et Ernest W. Burgess. Après avoir indiqué qu'il convient moins de parler d'« école » que de " tradition », Jean-Michel Chapoulie rappelle qu'elle constitue la première entreprise académique collective dans l'histoire des sciences sociales.

Revenant sur son propre parcours intellectuel, Howard Becker, qui fut l'un des principaux héritiers des méthodes d'enquête ethnographiques initiées par Park et Burgess et qui mêla études en sociologie et métier de jeune pianiste de jazz, peint dans un style impressionniste humoristique la vie nocturne des quartiers noirs de l'époque, sous toutes ses coutures.

\section{Champ culturel}

Dans une méditation sur «black », «bronze », and «blues » à partir de deux tableaux d'Archibald Motley représentant des scènes de rue de la vie nocturne de Bronzeville dans les années 1930, Richard Powell rapporte forme et contenu des toiles à la texture socio-économique des expériences de la rue. Il propose une brillante mise en rapport de conditions matérielles et de représentations autant artistiques que sociales, en traitant la rue comme performance. Le commerce visuel de l'espace urbain dans l'entre-deuxguerre est aussi au cœur des communications d'Henri Peretz et d'Amy Mooney, qui se concentrent sur la production photographique commerciale dans le South Side de Chicago jusque dans les années trente, en portant une attention particulière au studio Woodard. Ce corpus peu connu, qui échappe aux institutions culturelles et artistiques, montre la mise en place de nouveaux espaces publics visuels où des identités urbaine noire se construisent.

Dans une communication sur les productions littéraires et journalistiques de Richard Wright en réponse aux conditions sociales du ghetto de Chicago entre 1940 et 1951, Liesl Olson souligne le rapport dans ces travaux entre réalisme et modernisme, travail sur le terrain de Chicago et modernité internationale en lien avec Paris en particulier. L'un des axes du colloque est en effet l'étude du rapport entre construction d'institutions locales et rayonnement national ou international, Grande Migration vers Chicago et influence politique, culturelle et économique. Rebecca Zorach et Sarah Leboime présentent la scène picturale africaine-américaine de Chicago dans les années 1960 et 1970, et en particulier le groupe COBRA puis AfriCOBRA, dans ses rapports avec différents ailleurs; les deux communications s'attachent en effet aux rapports entre construction d'institutions locales et déplacements nationaux et internationaux, en Europe puis en Afrique autour d'un programme panafricain. Ces trajectoires artistiques et politiques, individuelles et collectives, et les ressources qu'elles produisent, sont 
envisagées dans leurs déterminations autant matérielles que symboliques, entre «in » et « out », local et global.

Alexandre Pierrepont revient sur l'histoire de l'Association for the Advancement of Creative Musicians fondée en 1965 à l'initiative du pianiste Muhal Richard Abrams. Dans une perspective "d'histoire du jazz par en bas ", l'anthropologue insiste sur la singularité de cette expérience collective dans l'histoire des pratiques artistiques occidentales: il s'agit d'un des rares exemples de production musicale d'avant-garde, expérimentale, hermétique, qui soit produite par des artistes issus des classes populaires, peu dotés en capitaux culturels et appartenant à des groupes sociaux marginalisés et opprimés. L'AACM est ainsi "moitié musique, moitié entraide » et s'inscrit dans un projet plus large de nationalisme culturel, d'auto-production et d'auto-gestion.

La communication de Forrest Stuart sur l'utilisation des réseaux sociaux et les pratiques culturelles des habitants dans les quartiers noirs, plus particulièrement des jeunes membres de gangs, rappelle en creux qu'une définition trop « légitimiste » du champ culturel conduit à exclure de l'analyse les pratiques culturelles des classes populaires. À partir d'un protocole d'enquête ethnographique original, mêlant enquête "en ligne» et "hors ligne», et loin des discours qui pathologisent les pratiques sociales, Stuart cherche à réinscrire ces pratiques culturelles dans une économie plus générale de biens matériels et symboliques tout en montrant que Chicago occupe une place centrale dans cette industrie culturelle en ligne. Pastichant une formule célèbre de l'ethnographe Elijah Anderson, Stuart parle ainsi d'un «code of the tweet » que les jeunes apprennent, utilisent, réécrivent et transgressent.

17 Le colloque aboutit le samedi après-midi à une série de dialogues entre sociologie, musique et peinture, entre récit individuel et collectif, et entre différents militants de l'éducation à Chicago des années soixante au présent. Ces différentes configurations forment des ponts entre disciplines, générations et époques, ponts qui sont l'aspect le plus original du colloque; ce dernier après-midi rassemble d'ailleurs des intervenants et un public qui replacent le travail académique dans une sphère publique plus large et plus diverse. L'artiste et directeur de la « Rebuild Foundation », Theaster Gates, clôt les trois jours de colloque par une conférence qui donne à voir certains rapports nouveaux entre artistique et économique (avec ses projets urbains, en particulier autour d'une banque transformée en centre - et capital - culturel), et aussi entre l'université de Chicago et les quartiers africains-américains du South Side au XXI ${ }^{\mathrm{e}}$ siècle.

\section{INDEX}

Thèmes : Actualité de la recherche 
AUTEURS

ELIANE DE LARMINAT

Paris Diderot-Paris VII

CLÉMENT PETITJEAN

Paris Sorbonne 